\title{
Efeito do teor de água e tamanho de partícula na decomposição térmica de pastas de cimento moídas
}

\author{
Effect of water content and particle size \\ on the thermal decomposition of \\ ground cement paste
}

\author{
Raquel Maria Rocha Oliveira Menezes ${ }^{1}$, Leonardo Martins Costa ${ }^{2}$, \\ Ludmila Rodrigues Costa Tavares ${ }^{1}$, Augusto Cesar da Silva Bezerra ${ }^{3}$, \\ Maria Teresa Paulino Aguilar ${ }^{2}$
}

\footnotetext{
${ }^{1}$ Departamento de Engenharia de Estruturas, Universidade Federal de Minas Gerais, Av. Antônio Carlos, 6627, CEP: 31270-901, Pampulha, Belo Horizonte, Minas Gerais, Brasil.

${ }^{2}$ Departamento de Engenharia de Materiais e Construção, Universidade Federal de Minas Gerais, Av. Antônio Carlos, 6627, CEP: 31270-901, Pampulha, Belo Horizonte, Minas Gerais, Brasil.

${ }^{3}$ Departamento de Engenharia de Transportes, Centro Federal de Educação Tecnológica de Minas Gerais, Av. Amazonas, 5.253, CEP: 30421-16, Nova Suíça, Belo Horizonte, Minas Gerais, Brasil.

e-mail: raq.oliveira.menezes@gmail.com, ludycosta@gmail.com, leonardocosta13@ufmg.br, teresa@ufmg.br augustobezerra@cefetmg.br
}

\section{RESUMO}

Um método alternativo para produção de concretos ecoeficientes seria a substituição parcial de cimento Portland por resíduos de pastas e argamassas submetidas a um tratamento termomecânico. Neste contexto, este estudo avalia a influência da relação água/cimento $(\mathrm{a} / \mathrm{c})$ e do tamanho médio das partículas na decomposição térmica do cimento hidratado. Pastas de cimento com três relações a/c $(0,40,0,50$ e 0,60$)$ e curadas por 28 dias foram moídas para obtenção de granulometrias com tamanho médio de 0,60 mm e 0,15 mm. A decomposição térmica das pastas foi analisada por termogravimetria (TGA), difração de raios X (DRX) e espectroscopia no infravermelho por Transformada de Fourier (FTIR). A análise de TGA indica que o teor de água quimicamente combinada é favorecido pelo aumento da relação a/c entre 0,40 e 0,50 , mas é constante para a/c de 0,50 e 0,60. A redução do tamanho médio quase não interfere nas curvas de TGA. De acordo com as análises por DRX e TGA, a maior parte dos hidratos se decompõe até $500-550^{\circ} \mathrm{C}$, enquanto que silicatos de cálcio e aluminatos de cálcio similares às fases anidras do cimento Portland foram detectados na pasta aquecida a $750^{\circ} \mathrm{C}$. Assim, temperaturas mais altas seriam necessárias para a completa recuperação do cimento anidro em pastas hidratadas.

Palavras-chave: Pasta de cimento Portland, decomposição térmica, relação água/cimento, tamanho de partículas, análises térmicas.

\section{ABSTRACT}

A possible method for producing eco-efficient concrete would be the partial replacement of Portland cement by pastes and mortars residues subjected to thermal-mechanic treatment. In this context, this study evaluates the influence of water/cement (w/c) ratio and average particle size on the thermal decomposition of hydrated cement. Cement pastes with three w/c ratios $(0.40,0.50$ and 0.60$)$ and cured by 28 days were ground to obtain two particle distributions with average sizes of $0.60 \mathrm{~mm}$ and $0.15 \mathrm{~mm}$. The thermal decomposition of pastes was analysed by thermal gravimetric analysis (TGA), X-ray diffraction (XRD) and Fourier transform infrared spectroscopy (FTIR). TGA analysis indicates that chemically bounded water content is improved by the increasing of w/c ratio between 0.40 and 0.50 , but it is constant between w/c of 0.50 and 0.60 . Particle size reduction almost does not interfere with TGA curves. According to XRD e TGA analysis, most hydrates are decomposed at $500-550{ }^{\circ} \mathrm{C}$, while calcium silicates and calcium aluminates similar to the anhydrous phases of Portland cement were detected on the heated paste at $750{ }^{\circ} \mathrm{C}$. Thus, higher temperatures would be 
required for the complete recovery of the anhydrous cement in hydrated pastes.

Keywords: Portland cement paste, thermal decomposition, water/cement ratio, particle size, thermal analysis.

\section{INTRODUÇÃO}

Um material cimentício suplementar pode ser obtido pelo tratamento termomecânico de resíduos de pastas e argamassas produzidas com agregado quartzoso [1-3]. Dessa forma é possível desidratar e recuperar o cimento [4-6] e produzir um pó rico em quartzo [7]. Este quartzo finamente moído pode apresentar elevada atividade pozolânica ou interagir fisicamente com o cimento hidratado por meio do preenchimento de vazios (efeito fíler) [3, 6, 8-13]. MENEZES et al. [3] obtiveram pó de quartzo com alto índice de pozolanicidade de acordo com o método Chapelle Modificado. No estudo de BENEZET e BENHASSAINE [7], pós de quartzos com diâmetro crítico abaixo de $5 \mu \mathrm{m}$ apresentaram elevada atividade pozolânica. BERODIER e SCRIVENER [11] e KUMAR et al. [13] indicaram que o uso de pó de quartzo estimula fisicamente a hidratação do cimento nas primeiras horas de cura. Contudo, KADRI et al. [9] concluíram que pó de quartzo acelera a hidratação do cimento de maneira pouco significativa em comparação com fíler calcário e sílica ativa. LAWRENCE et al. [10] notaram que a substituição parcial de cimento por pó de quartzo reduziu o calor de hidratação nas primeiras horas de cura, porém aumentou o grau de hidratação do cimento em idades avançadas.

Ao mesmo tempo, muitos autores têm estudado as fases formadas pela hidratação, desidratação e reidratação de pastas de cimento [4, 14-17]. Análises por termogravimetria (TGA), difração de raios X (DRX), espectroscopia no infravermelho por transformada de Fourier (FTIR) e análise térmica diferencial (DTA) têm sido amplamente empregadas nestes estudos [5, 6, 18-22]. SHUI et al. [18] estimaram o grau de reidratação de cimento desidratado calculando a perda de água não-evaporável por TGA. SPLITTGERBER e MUELLER [4] utilizaram DRX para estudar a recuperação de fases do cimento anidro após aquecimento de pasta de cimento hidratado até $1400{ }^{\circ} \mathrm{C}$ e descobriram que as fases são recuperáveis quando temperaturas próximas às de clinquerização são atingidas. ZHANG e YE [6], utilizando TGA e análise quantitativa de DRX (Refinamento de Rietveld), concluíram que a decomposição térmica do C-S-H pode ser dividida em duas etapas: perda de água presente entre as camadas, até $400^{\circ} \mathrm{C}$, e perda de água quimicamente combinada no intervalo entre $500-1000{ }^{\circ} \mathrm{C}$, o que provoca a quebra das cadeias de silicatos e a consequente cristalização do C-S-H em silicato dicálcico.

ALARCON-RUIZ et al. [21] verificaram, por meio de TGA, que pastas de cimento pré-aquecidas em temperaturas acima de $500{ }^{\circ} \mathrm{C}$ preservam teores residuais de água quimicamente combinada aos hidratos. De acordo com ALONSO e FERNANDEZ [19], a decomposição térmica completa do C-S-H em silicatos de cálcio de composição similar à belita (silicato dicálcico) ou alita (silicato tricálcico) ocorre em $750{ }^{\circ} \mathrm{C}$. SONG et al. [5], com base em análises por TGA e DRX em alta temperatura (HT-DRX), indicaram que etringita e monossulfato se desidratam completamente até $91{ }^{\circ} \mathrm{C}$ e $160{ }^{\circ} \mathrm{C}$, respectivamente, e que a maior parte da desidratação do C-S-H ocorre no intervalo de $80-240{ }^{\circ} \mathrm{C}$, enquanto que a decomposição das cadeias de silicatos de C-S-H ocorre em torno de 615-630 ${ }^{\circ} \mathrm{C}$. CHOUDHARY et al. [16] e BIRICIK e SARIER [22] utilizaram FTIR para avaliar a formação de fases hidratadas e não hidratadas em pastas de cimento, contudo, esta técnica ainda é pouco empregada para análise de decomposição térmica ou reidratação das pastas [23].

$\mathrm{O}$ aumento da temperatura de desidratação do cimento tende a aumentar o teor de água requerida na consistência normal e a reduzir consideravelmente o tempo de pega das pastas de cimento reidratado [18, 24]. SHUI et al. [18] mostraram que as propriedades cimentantes do cimento desidratado dependem da temperatura de desidratação. Os autores verificaram que pastas confeccionadas com o cimento desidratado a $800{ }^{\circ} \mathrm{C}$ tendem a desenvolver alta resistência mecânica inicial, porém com resistência aos 28 dias inferior à pasta confeccionada com o cimento anidro convencional. De acordo com SERPELL e LOPEZ [2], temperaturas de desidratação em $800{ }^{\circ} \mathrm{C}$ propiciaram maiores valores de resistência mecânica em compósitos cimentícios contendo resíduos de pasta de cimento desidratado. Os autores indicaram que substituição de cimento por até $35 \%$ de sílica ativa aumentou a resistência à compressão aos 28 dias de cura.

XUAN e SHUI [15] confeccionaram pastas com diferentes relações água/cimento (a/c) e, após tratamento térmico, os cimentos obtidos foram reidratados. Os autores concluíram que o aumento da relação água/cimento $(\mathrm{a} / \mathrm{c})$ das pastas submetidas ao aquecimento reduziu a resistência à compressão, diminuiu o tempo de pega e aumentou a demanda de água na consistência normal das pastas após a reidratação. BORDY et al. [23], por sua vez, estudaram a reciclagem de resíduos de pastas de cimento submetidos a moagem sem tratamento térmico como substituição parcial em cimento. Os autores concluíram que o cimento hidratado reciclado não propiciou efeito fíler e sítios de nucleação suplementares nos compósitos cimentícios e que a contribuição desse resíduo foi devido à reatividade das suas fases anidras.

Neste contexto, análises da hidratação e decomposição térmica de pastas de cimento são necessárias 
em pesquisas que proponham a reutilização de resíduos de construção com altos teores de cimento hidratado. Em especial, poucos estudos abordam a influência do tamanho médio das pastas de cimento moídas nos resultados obtidos por TGA [5]. Assim, este artigo avalia a influência da relação a/c e do tamanho médio de partículas originadas da moagem na decomposição térmica de pastas de cimento. Pastas de cimento com três distintas relações a/c foram submetidas a moagem após a cura, a fim de obter amostras com diâmetros médios de $0,60 \mathrm{~mm}$ e $0,15 \mathrm{~mm}$. A hidratação e decomposição térmica das pastas foram avaliadas por meio de TGA, DTA, DRX e FTIR.

\section{MATERIAIS E MÉTODOS}

As pastas foram confeccionadas com cimento Portland de alta resistência inicial e água com três relações água/cimento: $0,60,0,50$ e 0,40. As características físicas e químicas do cimento são apresentadas nas Tabelas 1 e 2 .

Tabela 1: Caracterização física do cimento Portland.

\begin{tabular}{l|l|c}
\hline \multicolumn{2}{l|}{ PROPRIEDADES } & VALORES \\
\hline \multirow{2}{*}{ Tempo de pega (min.) } & Inicial & 131 \\
\cline { 2 - 3 } & Final & 185 \\
\hline \multirow{2}{*}{ Perda de ignição $(\%)$} & $500{ }^{\circ} \mathrm{C}$ & 0,7 \\
\cline { 2 - 3 } & $1000^{\circ} \mathrm{C}$ & 3,5 \\
\hline \multicolumn{2}{l}{ Resíduo insolúvel $(\%)$} & 0,8 \\
\hline \multicolumn{2}{l}{ Permeabilidade de Blaine $\left(\mathrm{cm}^{2} / \mathrm{g}\right)$} & 4,765 \\
\hline \multicolumn{2}{l}{ Superfície específica $\left(\mathrm{m}^{2} / \mathrm{kg}\right)$} & 470,8 \\
\hline \multicolumn{2}{l}{ Massa específica $\left(\mathrm{g} / \mathrm{cm}^{3}\right)$} & 3,11 \\
\hline \multirow{2}{*}{$\begin{array}{l}\text { Resistência à compressão } \\
(\mathrm{MPa})\end{array}$} & 3 dias & 30 \\
\cline { 2 - 3 } & 28 dias & 55 \\
\hline
\end{tabular}

Tabela 2: Composição química do cimento Portland.

\begin{tabular}{c|c|c|c|c|c|c|c}
\hline $\mathrm{SiO}_{2}(\%)$ & $\mathrm{Al}_{2} \mathrm{O}_{3}(\%)$ & $\mathrm{CaO}(\%)$ & $\mathrm{Fe}_{2} \mathrm{O}_{3}(\%)$ & $\mathrm{MgO}(\%)$ & $\mathrm{SO}_{3}(\%)$ & $\mathrm{CO}_{2}(\%)$ & $\mathrm{K}_{2} \mathrm{O}(\%)$ \\
\hline 19,0 & 5,0 & 63,3 & 3,0 & 0,7 & 3,0 & 3,0 & 0,8 \\
\hline
\end{tabular}

As pastas foram preparadas usando um misturador mecânico de argamassa e transferidas para fôrmas metálicas cilíndricas de $50 \mathrm{~mm}$ de diâmetro por $100 \mathrm{~mm}$ de altura. Então, as amostras foram desmoldadas e curadas em ambiente saturado até os 28 dias. A influência da relação a/c na composição do cimento hidratado foi primeiramente avaliada por difração de raios X (DRX) em um difratômetro Philips-PANalytical PW 1710 com radiação $\mathrm{CuK} \alpha$, tensão de $40 \mathrm{kV}$, corrente elétrica de $30 \mathrm{~mA}$ e monocromador de cristal de grafite. A varredura variou entre $3,03-89,97^{\circ} 2 \theta$ com passo de $0,06^{\circ}$ por segundo. Em seguida, as pastas foram aquecidas $900{ }^{\circ} \mathrm{C}$ durante 60 minutos, usando uma taxa de aquecimento de $10{ }^{\circ} \mathrm{C} / \mathrm{min}$. As amostras aquecidas foram analisadas por espectroscopia no infravermelho por transformada de Fourier (FTIR). Os padrões de FTIR foram obtidos usando um espectrofotômetro Nicolet Thermo Scientific 380 com cristal de seleneto de zinco ( $\mathrm{ZnSe}$ ) com espectro entre 400 e $4000 \mathrm{~cm}^{-1}$, resolução de $4 \mathrm{~cm}^{-1}$ e 32 varreduras.

As pastas de cimento hidratado com relação a/c de 0,60, 0,50 e 0,40 também foram submetidas a moagem para obter dois tipos de amostras com diâmetros médios de 0,60 e 0,15 mm. A moagem foi executada em moinho planetário de alta-energia (Planetary Mill Pulverisette 5) na velocidade de $300 \mathrm{rpm}$, usando cápsulas e esferas de óxido de zircônio. Foram, então, produzidos seis tipos de pastas (Tabela 3).

Tabela 3: Identificação e caracterização das pastas.

\begin{tabular}{c|c|c}
\hline IDENTIFICAÇÃO & RELAÇÃO A/C & DIÂMETRO MÉDIO (MM) \\
\hline Pasta 1 & 0,60 & 0,60 \\
\hline Pasta 2 & 0,60 & 0,15 \\
\hline
\end{tabular}




\begin{tabular}{c|c|c}
\hline Pasta 3 & 0,50 & 0,60 \\
\hline Pasta 4 & 0,50 & 0,15 \\
\hline Pasta 5 & 0,40 & 0,60 \\
\hline Pasta 6 & 0,40 & 0,15 \\
\hline
\end{tabular}

As amostras moídas foram submetidas a análise térmica por termogravimetria (TGA), a sua derivada primeira (DTG) e análise térmica diferencial (DTA) a fim de avaliar a influência do tamanho médio de grãos e relação a/c na decomposição térmica do cimento hidratado. Utilizou-se o instrumento Shimadzu DTG - 6OH com atmosfera em ar e aquecimento na taxa de $10{ }^{\circ} \mathrm{C} / \mathrm{min}$ até $750{ }^{\circ} \mathrm{C}$

Em função dos resultados obtidos, a pasta com relação a/c de 0,50 e diâmetro médio de 0,15 mm (pasta 4) também foi aquecida nas temperaturas de 200, 500, 550, 700 e $750{ }^{\circ} \mathrm{C}$, sob taxa de aquecimento de $10{ }^{\circ} \mathrm{C} / \mathrm{min}$. Então, esta pasta foi analisada por DRX, usando o equipamento e condições previamente descritos, com o objetivo de estimar a temperatura adequada para remover a água quimicamente combinada e desidratar os compostos de cimento. Em cada método utilizado (TGA, DTA, DRX e FTIR), foi feita uma análise por amostra.

\section{RESULTADOS}

\subsection{Análise de DRX do cimento hidratado}

A Figura 1 mostra os padrões de DRX das pastas com relação a/c de 0,40, 0,50 e 0,60 curadas por 28 dias. Silicato de cálcio hidratado (C-S-H), sulfoaluminato de cálcio hidratado (etringita), hidróxido de cálcio e carbonato de cálcio são identificados em todas as pastas[17, 25]. O pico localizado próximo a $32^{\circ} 2 \theta$ pode ser resultado de uma sobreposição de picos associados ao C-S-H [26, 27] e aos silicatos tricálcico e dicálcico anidros [17, 28, 29]. O halo difuso em torno de $29^{\circ} 2 \theta$, típico de C-S-H nanocristalino [30], é igualmente discreto nas três amostras. Estes resultados reforçam que o uso de diferentes relações a/c não altera os principais componentes do cimento hidratado.

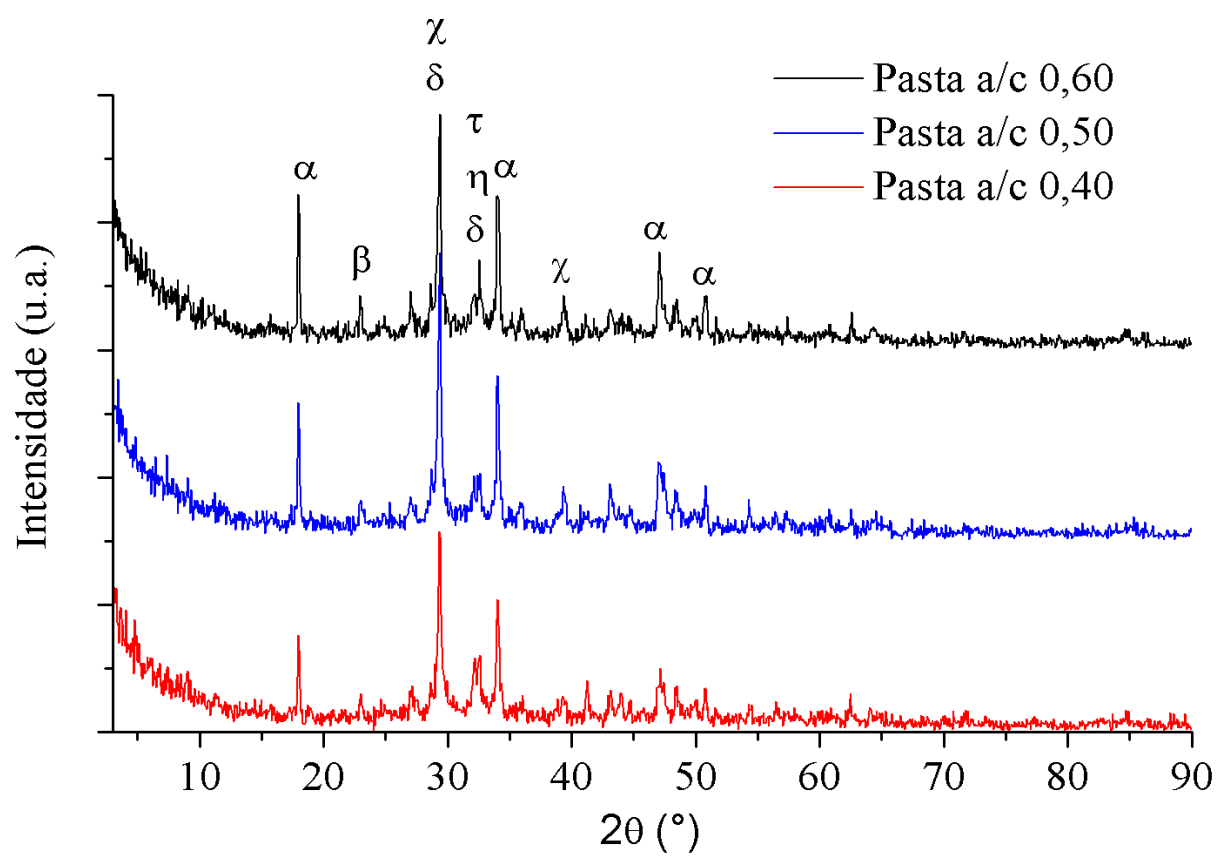

Figura 1: Difração de raios $X$ das pastas com diferentes relações a/c após 28 dias de cura; $\alpha$ - hidróxido de cálcio; $\beta$ etringita; $\chi$ - carbonato de cálcio; $\delta$ - silicato de cálcio hidratado $(\mathrm{C}-\mathrm{S}-\mathrm{H}) ; \tau$ - silicato tricálcico; e $\eta$ - silicato dicálcico.

\subsection{Análise de FTIR das pastas desidratadas}

Os resultados de FTIR para as amostras com diferentes relações a/c, aquecidas a $900{ }^{\circ} \mathrm{C}$, são mostrados na Figura 2. As bandas associadas às vibrações de grupos - $\mathrm{OH}$ de compostos hidratados e água livre em 3640 $\mathrm{cm}^{-1}, 3396 \mathrm{~cm}^{-1}$ e $1653 \mathrm{~cm}^{-1}$ [22,31] são detectadas em baixa intensidade, o que indica a decomposição dos 
hidratos durante o aquecimento.

A banda em $1409 \mathrm{~cm}^{-1}$ é relacionada ao estiramento de ligações CO em carbonatos de cálcio e a banda em $874 \mathrm{~cm}^{-1}$ é devida à vibração fora do plano de ligações $\mathrm{CO}$ em carbonatos ou ao estiramento de ligações SO em sulfatos $[16,32]$. A presença de silicato dicálcico anidro e silicato tricálcico é identificada nas bandas intensas em $964 \mathrm{~cm}^{-1}$ e $711 \mathrm{~cm}^{-1}$, relacionadas com o dobramento no plano ou estiramento de ligações Si-O $[22,23,32]$. A banda em $711 \mathrm{~cm}^{-1}$ também pode ser devida ao dobramento de ligações $\mathrm{CO}$ em carbonatos [32]. A elevada intensidade desses picos mostra que houve decomposição térmica de C-S-H em silicatos de cálcio anidros [5, 6]. Estes silicatos de cálcio apresentam composição química similar aos componentes majoritários do cimento Portland anidro $[4,19] \mathrm{e}$, portanto, o aquecimento até $900^{\circ} \mathrm{C}$ é suficiente para provocar a desidratação das pastas.

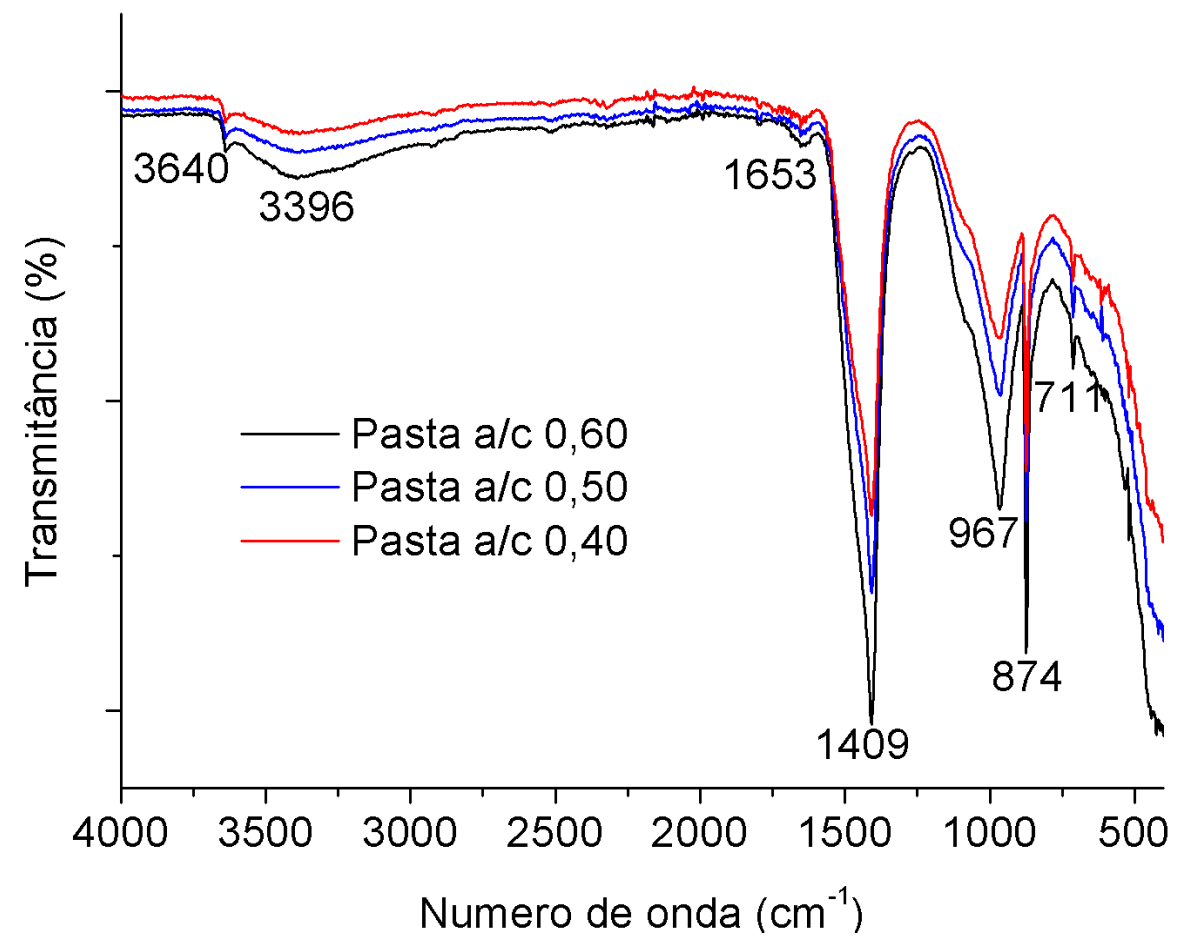

Figura 2: Análise de FTIR executada nas pastas aquecidas a $900^{\circ} \mathrm{C}$.

\subsection{Análise da decomposição térmica das pastas}

As Figuras 3 a 5 mostram os resultados da análise térmica das pastas de 1 a 6 . As curvas mostram os resultados obtidos por TGA, DTG e DTA, que são similares às curvas descritas na literatura para cimento Portland convencional $[5,12,17,33]$. Nestas figuras, são observadas três regiões distintas com perdas de massa significativas. A perda de massa na primeira região, entre a temperatura ambiente até $200{ }^{\circ} \mathrm{C}$, corresponde a evaporação da água livre, água adsorvida e a decomposição do C-S-H e etringita [5, 34]. A segunda região, no intervalo entre $400-480^{\circ} \mathrm{C}$, é resultado da decomposição do hidróxido de cálcio [12, 35]. No intervalo de $600-750{ }^{\circ} \mathrm{C}$, a decomposição do carbonato de cálcio é observada [17,33]. Os resultados obtidos sugerem que, até $500{ }^{\circ} \mathrm{C}$, há perda quase completa de água quimicamente combinada aos hidratos, mas que teores de compósitos hidratados podem ter resistido à decomposição até temperaturas mais elevadas [21]. 


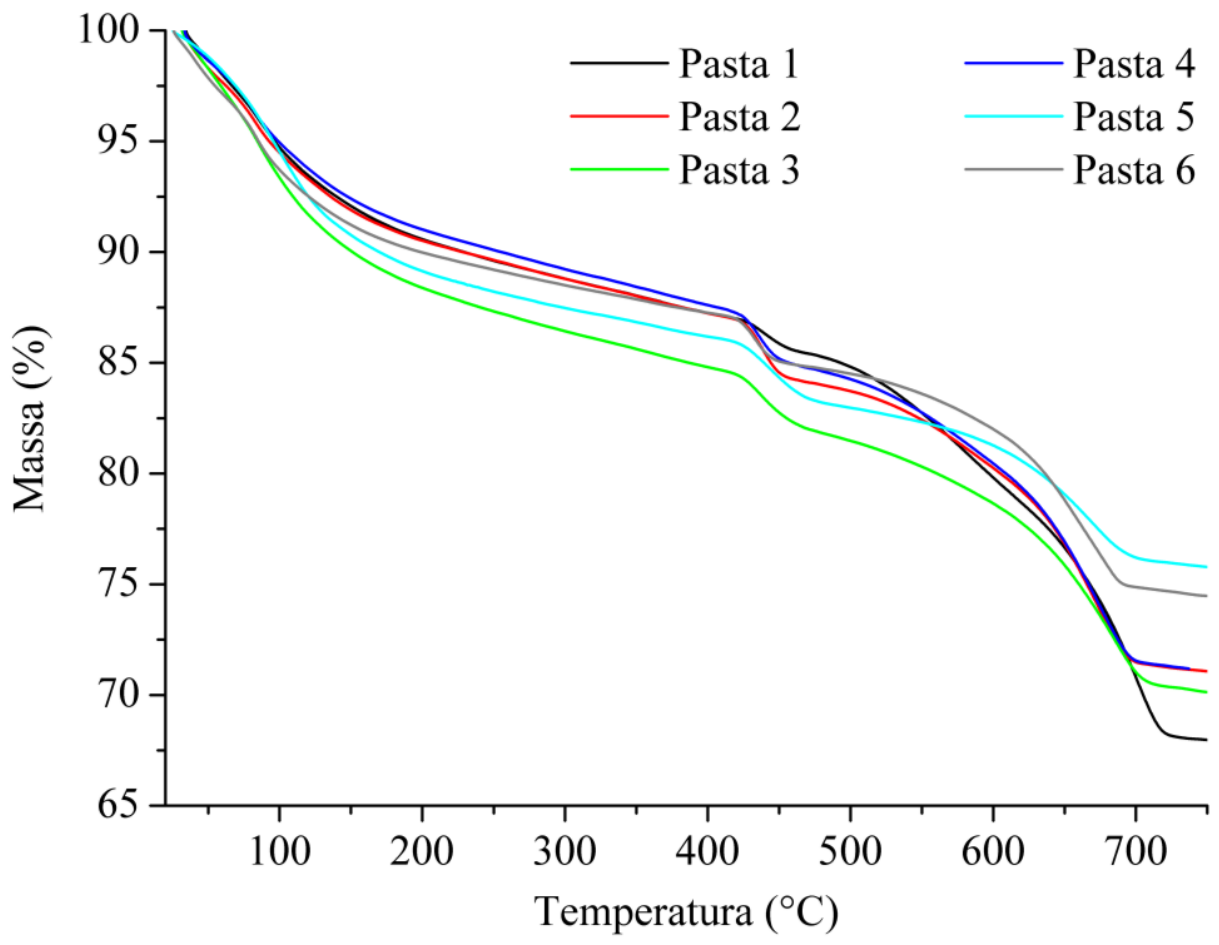

Figura 3: Curvas de TGA das pastas.

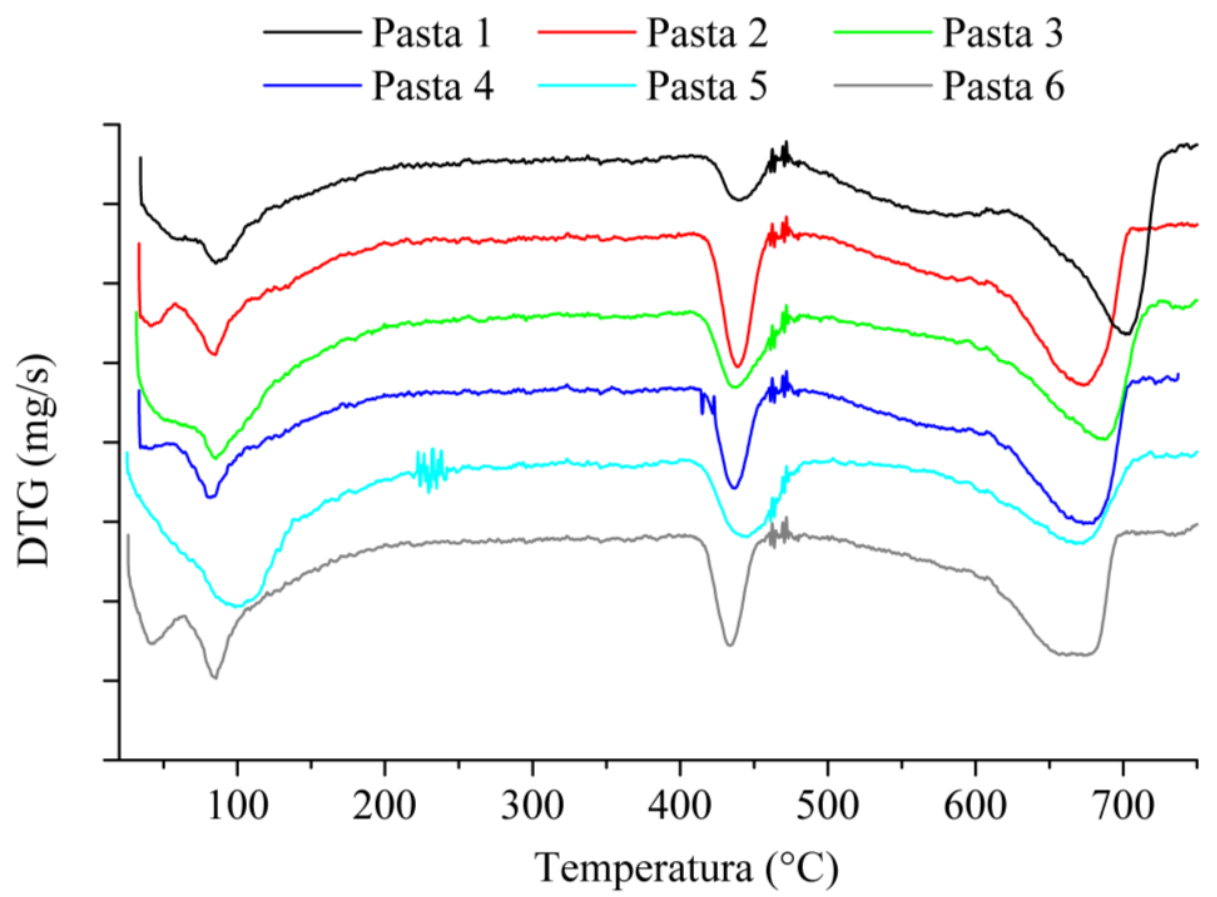

Figura 4: Curvas de DTG das pastas. 


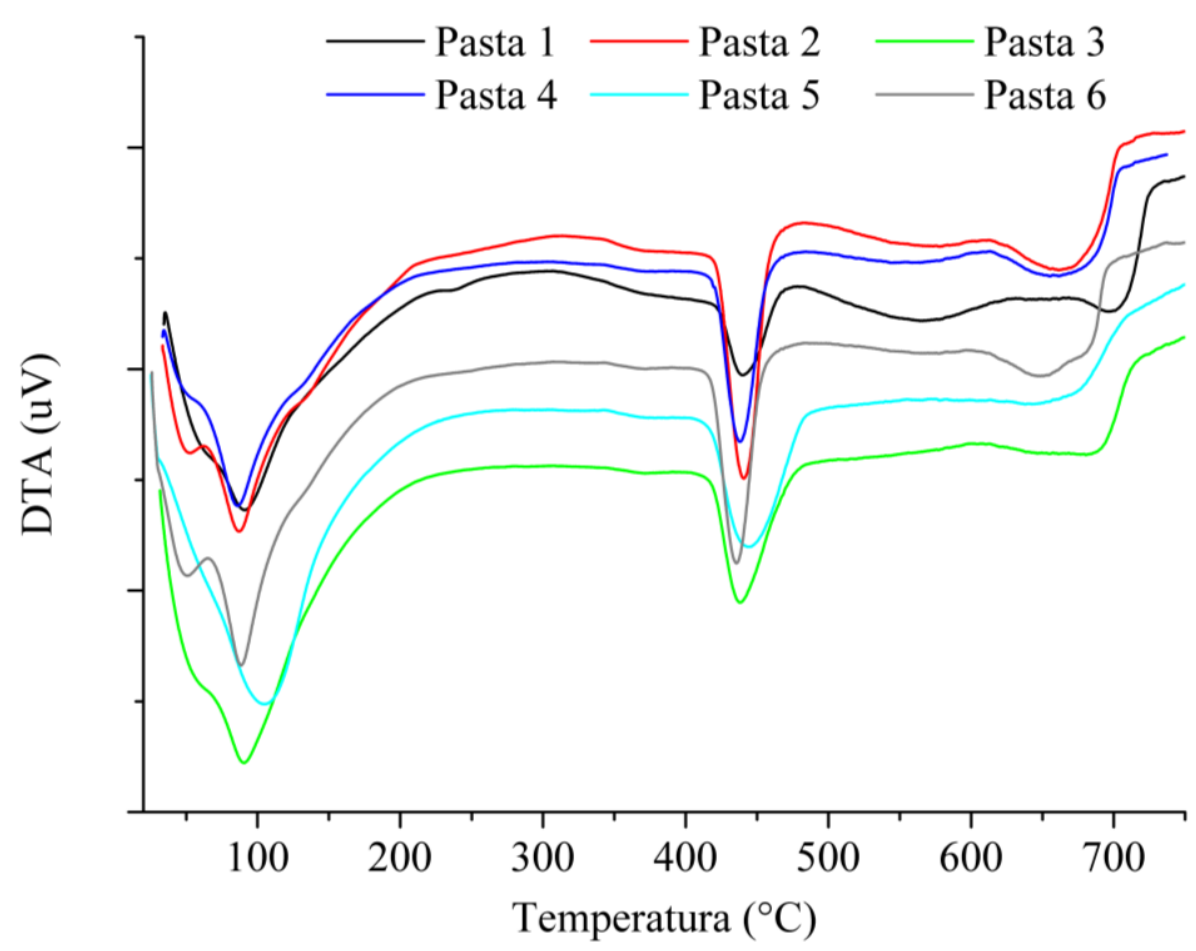

Figura 5: Análise de DTA das pastas.

Com base nas curvas de TGA, as perdas de massa relacionadas à decomposição de C-S-H, etringita e outros hidratados $\left(\mathrm{W}_{\text {hidratos }}\right)$, desidroxilação do hidróxido de cálcio $\left(\mathrm{W}_{\mathrm{CH}}\right)$ e decomposição de carbonato de cálcio $\left(\mathrm{W}_{\mathrm{CO}}\right)$ foram estimadas por meio das equações 1,2 e 3 , respectivamente $[17,36]$ :

$$
\begin{aligned}
& W_{\text {hidratos }}=\frac{\left(m_{100}-m_{400}\right)}{m_{f}} \times 100 \% \\
& W_{C H}=\frac{\left(m_{400}-m_{480}\right)}{m_{f}} \times 100 \% \\
& W_{C O}=\frac{\left(m_{750}-m_{600}\right)}{m_{f}} \times 100 \%
\end{aligned}
$$

Nestas equações, $\mathrm{m}_{100}$ é a massa das pastas $(\%)$ em $100^{\circ} \mathrm{C}, \mathrm{m}_{400}-\mathrm{m}_{480}$ e $\mathrm{m}_{750}-\mathrm{m}_{600}$ são os teores de perda de massa entre $400-480^{\circ} \mathrm{C}$ e $750-480^{\circ} \mathrm{C}$, respectivamente, e $\mathrm{m}_{\mathrm{f}}$ é a massa final (\%) das amostras. Considerando que a carbonatação das amostras após a cura consumiu parte do hidróxido de cálcio disponível, o teor de $\mathrm{Ca}(\mathrm{OH})_{2}$ equivalente ao carbonato de cálcio foi calculado conforme RUPASINGHE et al. [17]. Este teor corrigido de $\mathrm{Ca}(\mathrm{OH})_{2}$ foi adicionado ao valor obtido pela equação 2 .

Por fim, o teor total de água quimicamente combinada $\left(\mathrm{W}_{\text {total }}\right)$ nas pastas de cimento foi estimada usando a equação 4:

$$
W_{\text {total }}=W_{\text {hidratos }}+W_{C H}
$$

$\mathrm{O}$ teor de $\mathrm{W}_{\text {total }}$ foi comparado com a massa teórica de água quimicamente combinada $\left(\mathrm{W}_{\square}\right)$ requerida para a hidratação do cimento, que normalmente é de 0,23 ou $23 \%$ da massa de cimento anidro [37, 38]. A razão $\mathrm{R}$ entre os valores de $\mathrm{W}_{\text {total }} \mathrm{e} \mathrm{W}_{\square}$, calculada pela equação 5, pode ser usada para estimar o grau de hidratação das pastas estudadas [17].

$$
R=\frac{W_{\text {total }}}{W_{\infty}}
$$


A Tabela 4 mostra os valores de $\mathrm{W}_{\text {total }}$ e $\mathrm{R}$. Todas as pastas apresentam valores de $\mathrm{R}$ inferiores a 1 , o que pode ser explicado de duas formas distintas. Em primeiro, o cimento não foi hidratado completamente até os 28 dias de cura [23], isto é, a precipitação e o crescimento dos núcleos de C-S-H e outros hidratos restringem o contato dos grãos de clínquer com a água livre da mistura e reduzem a taxa de hidratação do cimento [39]. Em segundo, é possível que parte da água quimicamente combinada não foi removida da pasta durante a decomposição térmica até $480^{\circ} \mathrm{C}$ [21], o que subestimaria o cálculo de R.

Tabela 4: Valores de $\mathrm{W}_{\text {total }}$ e $\mathrm{R}$ das pastas.

\begin{tabular}{c|c|c}
\hline PASTAS & $\mathbf{W}_{\text {TOTAL }}(\%)$ & VALOR DE R \\
\hline 1 & 20,5 & 0,89 \\
\hline 2 & 19,4 & 0,85 \\
\hline 3 & 21,0 & 0,91 \\
\hline 4 & 19,1 & 0,83 \\
\hline 5 & 17,7 & 0,77 \\
\hline 6 & 15,5 & 0,68 \\
\hline
\end{tabular}

A Figura 6 apresenta os valores de R em função da relação a/c e do tamanho de partículas das pastas. Os valores de $\mathrm{R}$ tendem a aumentar em função do acréscimo da relação a/c. A variação da relação a/c de 0,40 para 0,50 aumenta o valor de $\mathrm{R}$ em $22 \%$ nas pastas de $0,15 \mathrm{~mm}$ e em $18 \%$ nas pastas de $0,60 \mathrm{~mm}$. Por outro lado, o aumento da relação a/c de 0,50 para 0,60 pouco influenciou no valor de R: leve aumento de $2 \%$ na pasta de $0,15 \mathrm{~mm}$ e redução de apenas $2 \%$ na pasta de $0,60 \mathrm{~mm}$.

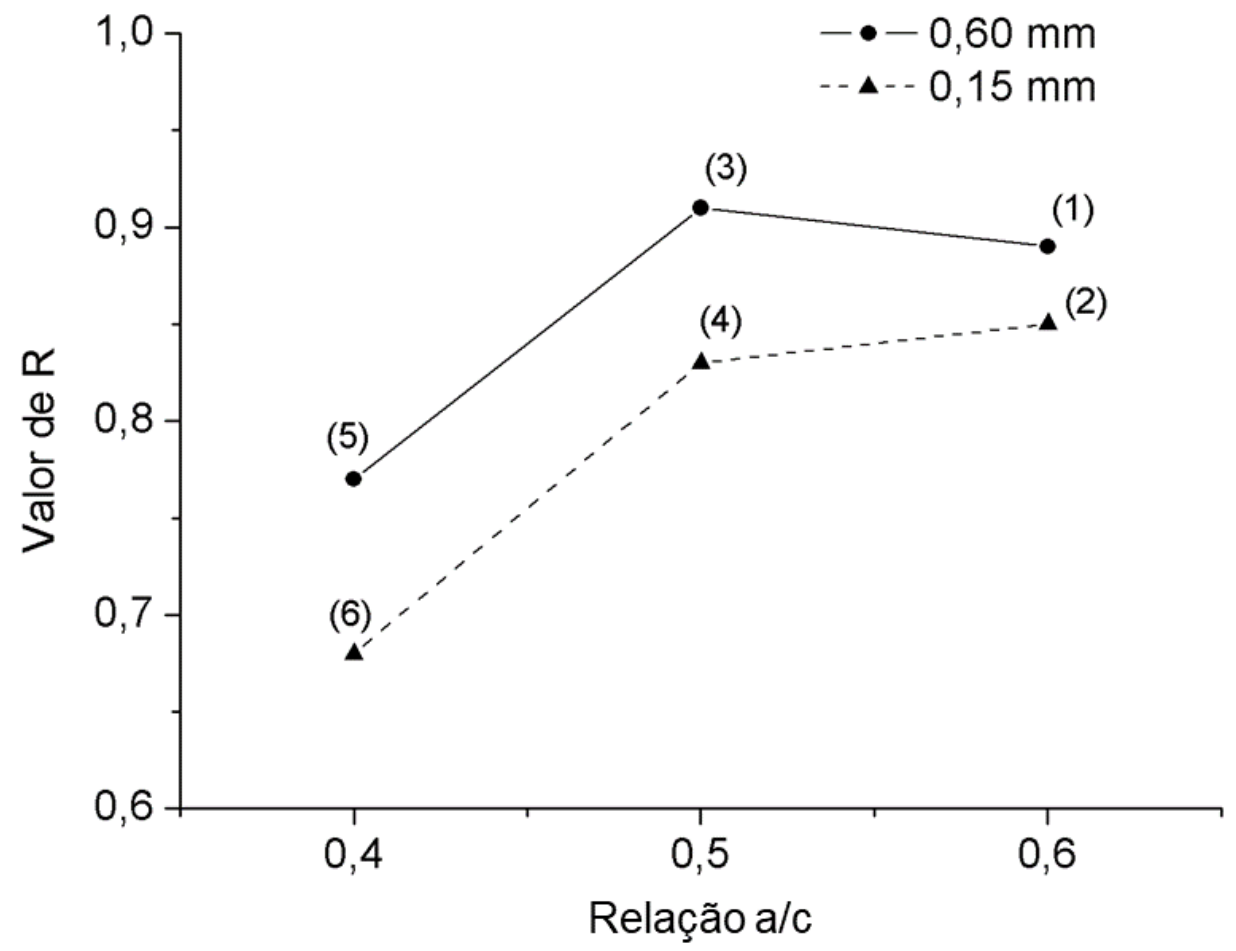

Figura 6: Valores de R em função da relação a/c e do diâmetro médio das pastas (as pastas correspondentes a cada ponto são indicadas entre parêntesis).

O volume disponível na mistura para o crescimento dos hidratos aumenta de acordo com o acréscimo da relação a/c, o que favorece a hidratação $[25,37]$ e, por conseguinte, intensifica o valor de R. No entanto, o aumento da relação a/c de 0,40 para 0,60 não é suficiente para que a hidratação do cimento seja completa aos 28 dias de cura. PANE e HANSEN [37] verificaram, por meio de calorimetria isotérmica, que o grau de hidratação de pasta de cimento com relação a/c de 0,45 foi inferior a 1,0 aos 28 dias de idade. BORDY et al. [23] utilizaram resíduos de cimento hidratado sem tratamento térmico na confecção de compósitos cimentícios e observaram que fases anidras desses resíduos sofreram hidratação durante a cura dos compósitos. Por- 
tanto, conforme a Figura 6, o teor de água quimicamente combinada detectado por TGA aumenta em função do acréscimo da relação a/c, porém, todas as amostras possuem valores de $\mathrm{R}$ inferiores a 1 , o que indica a hidratação incompleta do cimento aos 28 dias de cura.

Ademais, a redução do diâmetro médio das pastas de $0,60 \mathrm{~mm}$ para $0,15 \mathrm{~mm}$ devido à moagem diminui levemente os valores de $\mathrm{R}$ em todas as relações a/c estudadas, ou seja, a moagem das amostras após o tempo de cura exerce pouca influência nos valores de agua quimicamente combinada obtidos por TGA. Assim, a variação de distribuição granulométrica das pastas não propicia variação significativa na curva TGA, comportamento similar ao do estudo de SONG et al. [5], em que pastas de cimento de diâmetro mediano $44,79 \mu \mathrm{m}$ e $18,24 \mu \mathrm{m}$ apresentaram curvas TGA semelhantes.

\subsection{Análise de DRX das pastas aquecidas}

A Figura 7 mostra os espectros de DRX das pastas com relação a/c de 0,50 e tamanho médio de partículas de $0,15 \mathrm{~mm}$ (pasta 4), sem e com aquecimento nas temperaturas de $200,500,550,700$ e $750{ }^{\circ} \mathrm{C}$ [3]. A amostra não aquecida apresenta picos característicos de C-S-H, hidróxido de cálcio, etringita, calcita e silicatos tricálcico e dicálcico anidros $[17,25,26,29]$, conforme indicado no item 3.1. O aquecimento da pasta em $200{ }^{\circ} \mathrm{C}$ é suficiente para decompor completamente a etringita [5, 14, 35].

Uma mudança significativa na composição mineralógica é observada nas pastas aquecidas a partir de $500{ }^{\circ} \mathrm{C}$. Há uma redução significativa na intensidade dos picos de hidróxido de cálcio após $500^{\circ} \mathrm{C}$, devido à desidroxilação [6]. Porém, na pasta aquecida a $750{ }^{\circ} \mathrm{C}$, o pico detectado em torno de $18^{\circ} 2 \theta$ poderia estar relacionado à formação de uma nova fase [3] ou ser devido à presença de $\mathrm{Ca}(\mathrm{OH})_{2}$ residual, o que indicaria que a decomposição térmica da portlandita é parcialmente reversível [21]. Também, a presença de $\mathrm{Ca}(\mathrm{OH})_{2}$ residual seria outro indicativo de que parte dos compostos hidratados não é decomposta durante a análise térmica, o que implica em valores de $\mathrm{R}$ abaixo de 1, conforme previamente discutido no item 3.3.

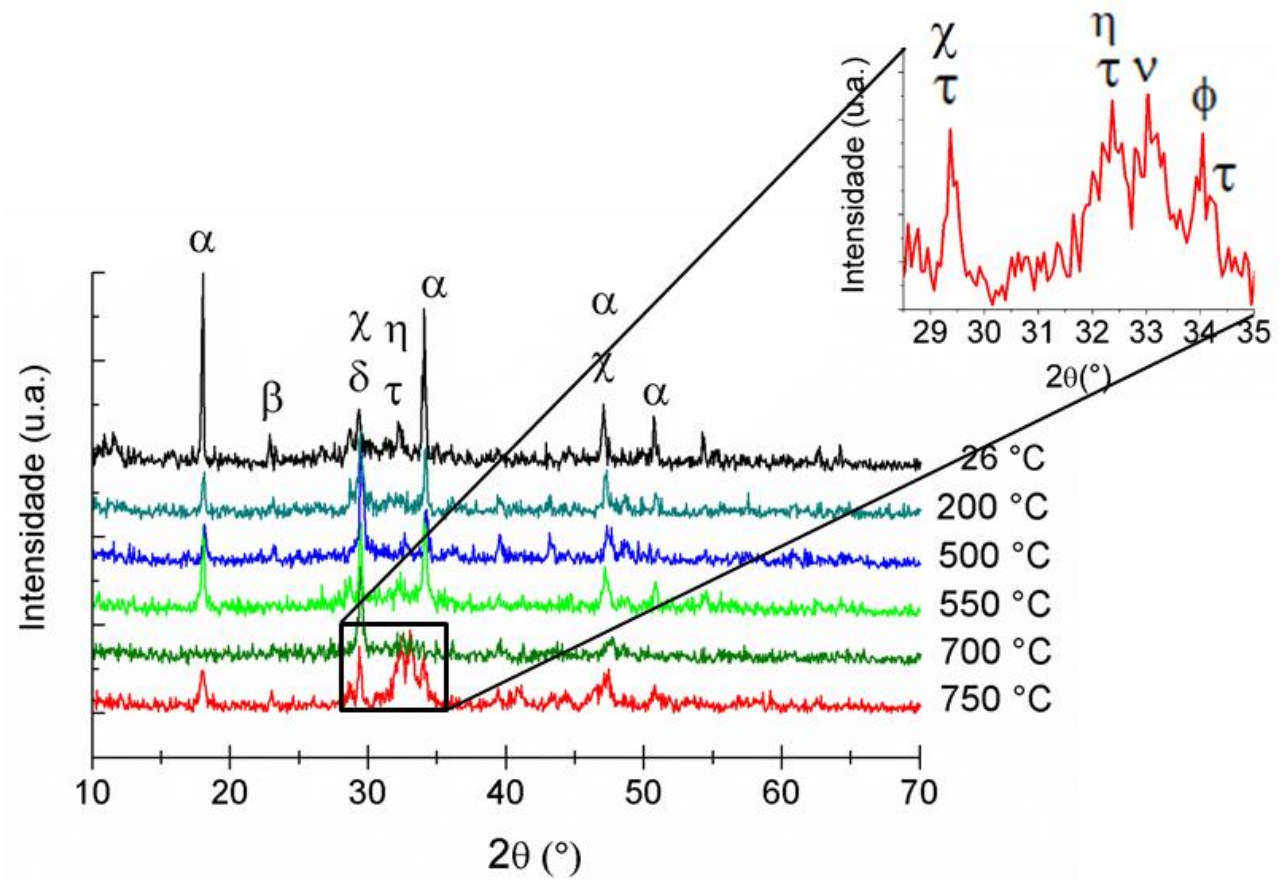

Figura 7: Espectros de DRX da pasta 4 mantida a $26^{\circ} \mathrm{C}$ e aquecida até $200,500,550,700$ e $750^{\circ} \mathrm{C}$; $\alpha$ - hidróxido de cálcio; $\beta$ - etringita; $\chi$ - carbonato de cálcio; $\delta$ - silicato de cálcio hidratado (C-S-H); $\eta$ - silicato dicálcico ; $\tau$ - silicato tricálcico; $v$ - aluminato tricálcico; e $\phi$ - ferro-aluminato de cálcio.

Por outro lado, ao contrário do observado por vários autores [2, 5, 6], nenhum pico de cal livre é detectado na Figura 7, o que pode ser explicado pela combinação de três fatores. O primeiro fator é que a portlandita desidroxilada pode preservar parcialmente a estrutura cristalina mesmo com a perda de água quimicamente combinada e, então, apresentar espectro de DRX similar ao $\mathrm{Ca}(\mathrm{OH})_{2}$ não decomposto [5]. Em segundo, a maior parte da cal formada pela desidroxilação do $\mathrm{Ca}(\mathrm{OH})_{2}$ pode ser amorfa ao DRX [6]. E terceiro, a cal formada pode ter sofrido carbonatação durante o armazenamento e preparo das amostras [16,17] ou ter 
reagido quimicamente com o carbono não oxidado presente no cimento [5]. Estas reações também explicariam a presença de picos de $\mathrm{CaCO}_{3}$ em temperaturas na ordem de $750^{\circ} \mathrm{C}$ e $900^{\circ} \mathrm{C}$ detectados por DRX e FTIR (Figura 2), respectivamente.

Ademais, na amostra aquecida a $750^{\circ} \mathrm{C}$, picos adicionais de silicatos dicálcico e tricálcico, aluminato de cálcio e ferroaluminato de cálcio são detectados no intervalo de 29-35² $2 \theta$ [2, 4-6], de maneira similar ao verificado no cimento Portland anidro. Isto mostra que a recuperação dos compostos anidros do cimento hidratado se inicia em temperaturas na ordem de $750^{\circ} \mathrm{C}$, o que poderia viabilizar a reutilização desse cimento como material de construção. A baixa intensidade dos picos relativos a esses compostos anidros, por outro lado, sugere que temperaturas mais altas podem ser necessárias para a desidratação satisfatória das pastas [4, $18]$.

\section{CONCLUSÕES}

Com base no estudo do efeito do teor de água e tamanho de partícula na decomposição térmica de pastas de cimento, conclui-se que:

1. As análises térmicas das pastas mostram que houve aumento do teor de água quimicamente combinada em função do acréscimo da relação a/c de 0,40 para 0,50, conforme indicado na Figura 6. Por outro lado, com o aumento da relação a/c de 0,50 para 0,60 , as variações no valor de $\mathrm{R}$ foram pouco significativas: leve aumento de $2 \%$ na pasta de $0,15 \mathrm{~mm}$ e redução de apenas $2 \%$ na pasta de $0,60 \mathrm{~mm}$. Contudo, como os valores estimados de $\mathrm{R}$ foram inferiores a 1 (Tabela 4) e fases anidras de cimento foram detectados na análise por DRX (Figura 1), as pastas com relações a/c de 0,40 a 0,60 sofreram hidratação incompleta aos 28 dias de cura. Ademais, é possível que parte da água quimicamente combinada não tenha sido removida, o que subestimaria a estimativa de $\mathrm{R}$.

2. A redução do tamanho médio das pastas de $0,60 \mathrm{~mm}$ para $0,15 \mathrm{~mm}$ pouco influenciou nas curvas de TGA e nos valores estimados de R, conforme indicado nas Figuras 3 e 6.

3. A análise de DRX das pastas aquecidas (Figura 7) indica que a maior parte da água quimicamente combinada é removida até $500-550{ }^{\circ} \mathrm{C}$, mas picos residuais de $\mathrm{Ca}(\mathrm{OH})_{2}$ se mantêm em temperaturas maiores. Silicatos de cálcio e aluminatos de cálcio de composição similar ao do cimento Portland são detectados na pasta aquecida em $750{ }^{\circ} \mathrm{C}$, porém com picos de baixa intensidade. Isto indica que temperaturas acima de $750{ }^{\circ} \mathrm{C}$ podem ser necessárias para a completa recuperação do cimento Portland anidro.

\section{AGRADECIMENTOS}

Os autores são gratos ao suporte financeiro do Conselho Nacional de Desenvolvimento Científica e Tecnológico (CNPq), Coordenação de Aperfeiçoamento de Pessoal de Nível Superior (CAPES) e Fundação de Amparo à Pesquisa do Estado de Minas Gerais (FAPEMIG).

\section{BIBLIOGRAFIA}

[1] SHUI, Z., XUAN, D., WAN, H., et al.., "Rehydration reactivity of recycled mortar from concrete waste experienced to thermal treatment", Construction and Building Materials, v. 22, n. 8, pp. 1723-1729, Jul. 2007.

[2] SERPELL, R., LOPEZ, M., "Properties of mortars produced with reactivated cementitious materials", Cement and Concrete Composites, v. 64, pp. 16-26, Set. 2015.

[3] MENEZES, R.M.R.O., DA SILVA, R.M., FIGUEIREDO, E.P., et al., "Hydraulic binder obtained from recycled cement and sand powder”, Revista Ibracon de Estruturas e Materiais, v. 11, n. 6, pp. 1178-1185, Dez. 2018.

[4] SPLITTGERBER, F., MUELLER, A., "Inversion of the Cement Hydration As a New Method for Identification and / or Recycling?", In: Proceedings of the 11th International Congress on the Chemistry of Cement, pp. 1282-1291, Durban, South Africa, Jan. 2003.

[5] SONG, H., JEONG, Y., BAE, S., et al., “A study of thermal decomposition of phases in cementitious systems using HT-XRD and TG”, Construction and Building Materials, v. 169, pp. 648-661, Mar. 2018.

[6] ZHANG, Q., YE, G., “Quantitative analysis of phase transition of heated Portland cement paste,” Journal of Thermal Analysis and Calorimetry, v. 112, pp. 629-636, Jul. 2012.

[7] BENEZET, J.C., BENHASSAINE, A., "The influence of particle size on the pozzolanic reactivity of quartz powders," Powder Technology, v. 103, pp. 26-29, Jun. 1999. 
[8] BENEZET, J.C., BENHASSAINE, A., "Contribuition of different granulometric populations to powder reactivity," Particuology, v. 7, pp. 39-44, Fev. 2009.

[9] KADRI, E.H., AGGOUN, S., DE SCHUTTER, G., et al., "Combined effect of chemical nature and fineness of mineral powders on Portland cement hydration," Materials and Structures, v. 43, n. 5, pp. 665-673, Jul. 2009.

[10] LAWRENCE, P., CYR, M., RINGOT, E., "Mineral admixtures in mortars: Effect of inert materials on short-term hydration," Cement and Concrete Research, v. 33, n. 12, pp. 1939-1947, Dez. 2003.

[11] BERODIER, E., SCRIVENER, K., "Understanding the filler effect on the nucleation and growth of CS-H," Journal of the American Ceramic Society, v. 97, n. 12, pp. 3764-3773, Ago. 2014.

[12] ANTONI, M., ROSSEN, J., MARTIRENA, F., et al., "Cement substitution by a combination of metakaolin and limestone," Cement and Concrete Research, v. 42, n. 12, pp. 1579-1589, Dez. 2012.

[13] KUMAR, A., OEY, T., FALZONE, G., et al., "The filler effect: The influence of filler content and type on the hydration rate of tricalcium silicate," Journal of the American Ceramic Society, v. 100, n. 7, pp. 33163328, Abr. 2017.

[14] FARAGE, M.C.R., SERCOMBE, J., GALLÉ, C., "Rehydration and microstructure of cement paste after heating at temperatures up to $300^{\circ} \mathrm{C}$, , Cement and Concrete Research, v. 33, n. 7, pp. 1047-1056, Jul. 2003.

[15] XUAN, D.X., SHUI, Z.H., "Rehydration activity of hydrated cement paste exposed to high temperature," Fire and Materials, v. 35, n. 9, pp. 481-490, Nov. 2010.

[16] CHOUDHARY, H. K., ANUPAMA, A. V., KUMAR, R., et al., "Observation of phase transformations in cement during hydration," Construction and Building Materials, v. 101, pp. 122-129, Dez. 2015.

[17] RUPASINGHE, M., SAN NICOLAS, R., MENDIS, P., et al., "Investigation of strength and hydration characteristics in nano-silica incorporated cement paste," Cement and Concrete Composites, v. 80, pp. 17-30, Fev. 2017.

[18] SHUI, Z., XUAN, D., CHEN, W., et al., "Cementitious characteristics of hydrated cement paste subjected to various dehydration temperatures," Construction and Building Materials, v. 23, n. 1, pp. 531-537, Dez. 2007.

[19] ALONSO, C., FERNANDEZ, L., "Dehydration and rehydration processes of cement paste exposed to high temperature environments," Journal of Materials Science, v. 39, n. 9, pp. 3015-3024, Mai. 2004.

[20] ROMANO, R.C.O., FUJII, A.L., SOUZA, R.B., et al., "Acompanhamento da hidratação de cimento Portland simples com resíduo de bauxita", Cerâmica, v. 62, n. 363, pp. 215-223, Set. 2016.

[21] ALARCON-RUIZ, L., PLATRET, G., MASSIEU, E., et al., "The use of thermal analysis in assessing the effect of temperature on a cement paste," Cement and Concrete Research, v. 35, n. 3, pp. 609-613, Mar. 2005.

[22] BIRICIK, H., SARIER, N., "Comparative study of the characteristics of nano silica - , silica fume - and fly ash - incorporated cement mortars," Materials Research, v. 17, n. 3, pp. 570-582, Mai. 2014.

[23] BORDY, A., YOUNSI, A., AGGOUN, S., et al., "Cement substitution by a recycled cement paste fine: Role of the residual anhydrous clinker", Construction and Building Materials, v. 132, pp. 1-8, Dez. 2016.

[24] VYŠVAŘIL, M., BAYER, P., CHROMÁ, M., et al., "Physico-mechanical and microstructural properties of rehydrated blended cement pastes," Construction and Building Materials, v. 54, pp. 413-420, Jan. 2014.

[25] LURA, P., WINNEFELD, F., FANG, X., "A simple method for determining the total amount of physically and chemically bound water of different cements," Journal of Thermal Analysis and Calorimetry, v. 130, n. 2, pp. 653-660, Jun. 2017.

[26] ROMANO, R.C.O., BERNARDO, H.M., MACIEL, M.H., et al., "Hydration of Portland cement with red mud as mineral addition," Journal of Thermal Analysis and Calorimetry, v. 131, n. 3, pp. 2477-2490, Nov. 2017.

[27] FILHO, J.H., GOBBI, A., PEREIRA, E., et al., "Atividade pozolânica de adições minerais para cimento portland (Parte II): índice de atividade pozolânica com cimento portland (IAP), difração de raios-X (DRX) e termogravimetria (TG/DTG)", Revista Matéria, v. 22, n. 3, Ago. 2017.

[28] YU, Q., SAWAYAMA, K., SUGITA, S., et al., "The reaction between rice husk ash and $\mathrm{Ca}(\mathrm{OH})_{2}$ solution and the nature of its product," Cement and Concrete Research, v. 29, n. 1, pp. 37-43, Jan. 1999. 
[29] GHODDOUSI, P., ADELZADE SAADABADI, L., "Study on hydration products by electrical resistivity for self-compacting concrete with silica fume and metakaolin," Construction and Building Materials, v. 154, pp. 219-228, Ago. 2017.

[30] SNELLINGS, R., SALZE, A., SCRIVENER, K.L., "Use of X-ray diffraction to quantify amorphous supplementary cementitious materials in anhydrous and hydrated blended cements," Cement and Concrete Research, v. 64, pp. 89-98, Jul. 2014.

[31] YLMÉN, R., JÄGLID, U., STEENARI, B.M., et al., "Early hydration and setting of Portland cement monitored by IR, SEM and Vicat techniques," Cement and Concrete Research, v. 39, n. 5, pp. 433-439, Mai. 2009.

[32] MENDES, A., GATES, W.P., SANJAYAN, J.G., et al., "NMR, XRD, IR and synchrotron NEXAFS spectroscopic studies of OPC and OPC/slag cement paste hydrates," Materials and Structures/Materiaux et Constructions, v. 44, n. 10, pp. 1773-1791, Abr. 2011.

[33] BADANOIU, A., GEORGESCU, M., PURI, A., "The study of 'DSP' binding systems by thermogravimetry and differential thermal analysis," Journal of Thermal Analysis and Calorimetry, v. 74, n. 1, pp. 6575, Out. 2003.

[34] NOCHAIYA, T., WONGKEO, W., CHAIPANICH, A., "Utilization of fly ash with silica fume and properties of Portland cement-fly ash-silica fume concrete," Fuel, v. 89, n. 3, pp. 768-774, Out. 2010.

[35] PAYA, J., MONZÓ, J., BORRACHERO, M. V., et al., "Determination of the pozzolanic activity of fluid catalytic cracking residue. Thermogravimetric analysis studies on FC3R - lime pastes", Cement and Concrete Research, v. 33, pp. 1085-1091, Jul. 2003.

[36] FRANKEOVÁ, D., SLÍŽKOVÁ, Z., "Determination of the pozzolanic activity of mortar's components by thermal analysis", Journal of Thermal Analysis and Calorimetry, v. 125, n. 3, pp. 1115-1123, Mar. 2016.

[37] PANE, I., HANSEN, W., "Investigation of blended cement hydration by isothermal calorimetry and thermal analysis", Cement and Concrete Research, v. 35, n. 6, pp. 1155-1164, Jun. 2005.

[38] JENSEN, O.M., HANSEN, P.F., "Water-entrained cement-based materials - I. Principles and theoretical background", Cement and Concrete Research, v. 31, n. 4, pp. 647-654, Abr. 2001.

[39] SCRIVENER, K.L., JUILLAND, P., MONTEIRO, P.J.M., "Advances in understanding hydration of Portland cement”, Cement and Concrete Research, v. 78, pp. 38-56, Jun. 2015.

\section{ORCID}

Raquel Maria Rocha Oliveira Menezes Leonardo Martins Costa Ludmila Rodrigues Costa Tavares Augusto Cesar da Silva Bezerra Maria Teresa Paulino Aguilar https://orcid.org/0000-0002-6567-3180

https://orcid.org/0000-0002-0656-649X

https://orcid.org/0000-0003-4952-6823

https://orcid.org/0000-0003-1670-2376

https://orcid.org/0000-0002-0121-0881 